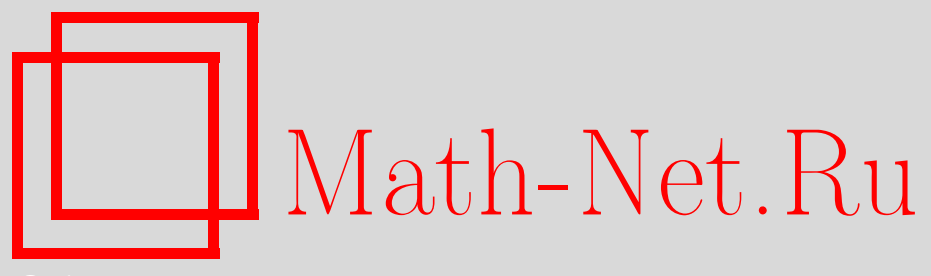

Сонгсиао Ли, С. Стевич, Дифференцирование суперпозиции как оператор из пространств со смешанной нормой в $\alpha$-пространства Блоха, Матем. сб., 2008, том 199, номер 12, 117-128

DOI: https://doi.org/10.4213/sm3794

Использование Общероссийского математического портала Math-Net.Ru подразумевает, что вы прочитали и согласны с пользовательским соглашением http://www . mathnet.ru/rus/agreement

Параметры загрузки:

IP: 18.209 .158 .208

26 апреля 2023 г., 12:40:47

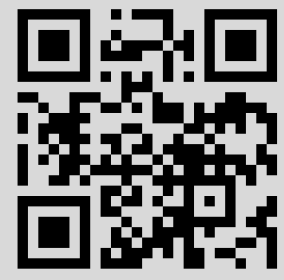




\section{Сонгсиао Ли, С. Стевич \\ Дифференцирование суперпозиции как оператор из пространств со смешанной нормой в $\alpha$-пространства Блоха}

Изучаются ограниченность и компактность произведения оператора дифференцирования и оператора суперпозиции, действующего из пространств со смешанной нормой в $\alpha$-пространства Блоха.

Библиография: 18 названий.

\section{§ 1. Введение}

Пусть $\mathbb{D}$ - открытый круг единичного радиуса в комплексной плоскости $\mathbb{C}$ и $H(\mathbb{D})$ - класс функций, аналитических в $\mathbb{D}$. Пространство со смешанной нормой $H_{p, q, \gamma}=H_{p, q, \gamma}(\mathbb{D}), 0<p, q<\infty,-1<\gamma<\infty$, состоит из тех функций $f \in H(\mathbb{D})$, для которых

$$
\|f\|_{H_{p, q, \gamma}}=\left(\int_{0}^{1} M_{p}^{q}(f, r)(1-r)^{\gamma} d r\right)^{1 / q}<\infty,
$$

где

$$
M_{p}(f, r)=\left(\frac{1}{2 \pi} \int_{0}^{2 \pi}\left|f\left(r e^{i \theta}\right)\right|^{p} d \theta\right)^{1 / p} .
$$

Согласно определению $f \in H(\mathbb{D})$ принадлежит $\alpha$-пространству Блоха $\mathscr{B}^{\alpha}=$ $\mathscr{B}^{\alpha}(\mathbb{D}), 0<\alpha<\infty$, если

$$
B_{\alpha}(f)=\sup _{z \in \mathbb{D}}\left(1-|z|^{2}\right)^{\alpha}\left|f^{\prime}(z)\right|<\infty
$$

Выражение $B_{\alpha}(f)$ задает полунорму, а естественная норма определяется формулой $\|f\|_{\mathscr{B}^{\alpha}}=|f(0)|+B_{\alpha}(f)$. $\mathrm{C}$ этой нормой $\mathscr{B}^{\alpha}$ является банаховым пространством. При $\alpha=1 \mathscr{B}^{1}=\mathscr{B}$ - хорошо известное пространство Блоха. Пусть $\mathscr{B}_{0}^{\alpha}-$ подпространство $\mathscr{B}^{\alpha}$, состоящее из функций $f \in \mathscr{B}^{\alpha}$, для которых

$$
\left(1-|z|^{2}\right)^{\alpha}\left|f^{\prime}(z)\right| \rightarrow 0, \quad|z| \rightarrow 1
$$

Оно называется малым $\alpha$-пространством Блоха.

Пусть $\varphi$ - аналитическое отображение $\mathbb{D}$ в себя. Сопоставим $\varphi$ оператор суперпозиции $C_{\varphi}$, определенный формулой

$$
C_{\varphi} f=f \circ \varphi, \quad f \in H(\mathbb{D}) .
$$

(C) Сонгсино Ли, С. Стевич, 2008 
Согласно хорошо известному следствию принципа подчинения Литтлвуда оператор суперпозиции $C_{\varphi}$ ограничен в классических пространствах Харди и Бергмана. Представляет интерес характеризация в терминах теории функций тех $\varphi$, которые порождают ограниченные или компактные операторы суперпозиции в тех или иных пространствах (см., например, [1]-[3] и ссылки в них).

Пусть $D$ - оператор дифференцирования. Оператор суперпозиции принадлежит к числу операторов, обычно являющихся ограниченными, а оператор дифференцирования, как правило, неограничен в различных пространствах аналитических функций. Произведение операторов суперпозиции и дифференцирования, оператор $D C_{\varphi}$, определяется по формуле

$$
D C_{\varphi}(f)=(f \circ \varphi)^{\prime}=f^{\prime}(\varphi) \varphi^{\prime}, \quad f \in H(\mathbb{D}) .
$$

Его впервые рассмотрели Хибшвайлер и Портной в статье [4], где исследовались ограниченность и компактность $D C_{\varphi}$ как оператора из пространства Харди в пространство Бергмана.

В настоящей работе изучается действие $D C_{\varphi}$ из пространств со смешанной нормой в $\alpha$-пространства Блоха. Приведены необходимые и достаточные условия ограниченности и компактности оператора $D C_{\varphi}$. Работу можно считать продолжением предыдущих исследований авторов, связанных с операторами в пространствах аналитических функций (см. [5]-[14]).

В тексте статьи все константы обозначены символом $C$, они всегда положительны и могут иметь различные значения в разных местах. Если для некоторых функций $A(\cdot)$ и $B(\cdot)$ существует такая постоянная $C>0$, что $B(\cdot) / C \leqslant A(\cdot) \leqslant C B(\cdot)$, то используется запись $A(\cdot) \asymp B(\cdot)$.

\section{§ 2. Ограниченность и компактность оператора $D C_{\varphi}: H_{p, q, \gamma} \rightarrow \mathscr{B}^{\alpha}$}

В этом параграфе мы охарактеризуем свойства ограниченности и компактности оператора $D C_{\varphi}: H_{p, q, \gamma} \rightarrow \mathscr{B}^{\alpha}$. Для доказательства основных результатов статьи потребуются следующие вспомогательные леммы.

Лемма 1. Пусть $0<p, q<\infty,-1<\gamma<\infty u f \in H_{p, q, \gamma}$. Тогда выполнено неравенство

$$
\left|f^{\prime}(z)\right| \leqslant C \frac{\|f\|_{H_{p, q, \gamma}}}{\left(1-|z|^{2}\right)^{(\gamma+1) / q+1 / p+1}}
$$

где $C>0$ - некоторая постоянная, не зависящая от $f$.

ДоКАЗАТЕЛЬСТво. В силу монотонности интегральных средних, используя хорошо известную оценку

$$
\int_{0}^{1} M_{p}^{q}(f, r)(1-r)^{\gamma} d r \asymp|f(0)|^{q}+\int_{0}^{1} M_{p}^{q}\left(f^{\prime}, r\right)(1-r)^{\gamma+q} d r
$$


(см., например, [15]) и теорему 7.2.5 в [16], получаем

$$
\begin{aligned}
\|f\|_{H_{p, q, \gamma}}^{q} & \geqslant \int_{(1+|z|) / 2}^{1} M_{p}^{q}\left(f^{\prime}, r\right)(1-r)^{\gamma+q} d r \geqslant C M_{p}^{q}\left(f^{\prime}, \frac{(1+|z|)}{2}\right)\left(1-|z|^{2}\right)^{\gamma+1+q} \\
& \geqslant C\left(1-|z|^{2}\right)^{\gamma+1+q+q / p}\left|f^{\prime}(z)\right|^{q}
\end{aligned}
$$

что и приводит к нужному результату.

Аналогичными рассуждениями с использованием оценки

$$
\int_{0}^{1} M_{p}^{q}(f, r)(1-r)^{\gamma} d r \asymp|f(0)|^{q}+\left|f^{\prime}(0)\right|^{q}+\int_{0}^{1} M_{p}^{q}\left(f^{\prime \prime}, r\right)(1-r)^{\gamma+2 q} d r
$$

доказывается следующая

Лемма 2. Пустъ $0<p, q<\infty,-1<\gamma<\infty u f \in H_{p, q, \gamma}$. Тогда

$$
\left|f^{\prime \prime}(z)\right| \leqslant C \frac{\|f\|_{H_{p, q, \gamma}}}{\left(1-|z|^{2}\right)^{(\gamma+1) / q+1 / p+2}}
$$

где $C>0$ - некоторая постоянная, не зависящая от $f$.

Следующая лемма приведена в [17].

ЛЕмма 3. При $\beta>-1, m>1+\beta$ выполнено неравенство

$$
\int_{0}^{1} \frac{(1-r)^{\beta}}{(1-\rho r)^{m}} d r \leqslant C(1-\rho)^{1+\beta-m}, \quad 0<\rho<1 .
$$

Следующий критерий компактности вытекает из стандартных соображений (см., например, предложение 3.11 в [1]).

Лемма 4. Пусть $0<p, q, \alpha<\infty,-1<\gamma<\infty u \varphi$-аналитическое отображение $\mathbb{D}$ в себя. Тогда $D C_{\varphi}: H_{p, q, \gamma} \rightarrow \mathscr{B}^{\alpha}$ - компактный оператор в том и только том случае, когда он ограничен и для всякой ограниченной последовательности $\left(f_{n}\right)_{n \in \mathbb{N}} \in H_{p, q, \gamma}$, сходящейся $\kappa$ нулю равномерно на компактных подмножествах $\mathbb{D}$, имеет место сходимость $\left\|D C_{\varphi} f_{n}\right\|_{\mathscr{B}^{\alpha}} \rightarrow 0, n \rightarrow \infty$.

Теперь сформулируем и докажем основные результаты этого параграфа.

Теорема 1. Пусть $0<p, q, \alpha<\infty,-1<\gamma<\infty u \varphi-$ аналитическое отображение $\mathbb{D}$ в себя. Тогда оператор $D C_{\varphi}: H_{p, q, \gamma} \rightarrow \mathscr{B}^{\alpha}$ ограничен в том и только том случае, когда выполнены следующие условия:

$$
\begin{aligned}
& \sup _{z \in \mathbb{D}} \frac{\left|\varphi^{\prime}(z)\right|^{2}\left(1-|z|^{2}\right)^{\alpha}}{\left(1-|\varphi(z)|^{2}\right)^{(\gamma+1) / q+1 / p+2}}<\infty, \\
& \sup _{z \in \mathbb{D}} \frac{\left|\varphi^{\prime \prime}(z)\right|\left(1-|z|^{2}\right)^{\alpha}}{\left(1-|\varphi(z)|^{2}\right)^{(\gamma+1) / q+1 / p+1}}<\infty .
\end{aligned}
$$


ДокАЗАТЕЛЬСтво. Предположим, что условия (3) и (4) выполнены. Тогда для всякого $z \in \mathbb{D}$ и для $f \in H_{p, q, \gamma}$ из (1) и (2) имеем

$$
\begin{aligned}
& \left(1-|z|^{2}\right)^{\alpha}\left|\left(D C_{\varphi} f\right)^{\prime}(z)\right| \leqslant\left(1-|z|^{2}\right)^{\alpha}\left|\left(f^{\prime}(\varphi) \varphi^{\prime}\right)^{\prime}(z)\right| \\
& \leqslant\left(1-|z|^{2}\right)^{\alpha}\left|\varphi^{\prime}(z)\right|^{2}\left|f^{\prime \prime}(\varphi(z))\right|+\left(1-|z|^{2}\right)^{\alpha}\left|\varphi^{\prime \prime}(z)\right|\left|f^{\prime}(\varphi(z))\right| \\
& \leqslant C \frac{\left|\varphi^{\prime}(z)\right|^{2}\left(1-|z|^{2}\right)^{\alpha}}{\left(1-|\varphi(z)|^{2}\right)^{(\gamma+1) / q+1 / p+2}}\|f\|_{H_{p, q, \gamma}} \\
& \quad+C \frac{\left|\varphi^{\prime \prime}(z)\right|\left(1-|z|^{2}\right)^{\alpha}}{\left(1-|\varphi(z)|^{2}\right)^{(\gamma+1) / q+1 / p+1}}\|f\|_{H_{p, q, \gamma}} .
\end{aligned}
$$

Взяв в (5) супремум по $z \in \mathbb{D}$ и используя условия (3) и (4), получим, что оператор $D C_{\varphi}: H_{p, q, \gamma} \rightarrow \mathscr{B}^{\alpha}$ ограничен.

Обратно, пусть $D C_{\varphi}: H_{p, q, \gamma} \rightarrow \mathscr{B}^{\alpha}$ ограничен, т.е. имеется постоянная $C$ такая, что

$$
\left\|D C_{\varphi} f\right\|_{\mathscr{B}^{\alpha}} \leqslant C\|f\|_{H_{p, q, \gamma}}, \quad f \in H_{p, q, \gamma} .
$$

Положив $f(z)=z$ и $f(z)=z^{2}$, получаем

$$
\begin{gathered}
\sup _{z \in \mathbb{D}}\left(1-|z|^{2}\right)^{\alpha}\left|\varphi^{\prime \prime}(z)\right|<\infty, \\
\sup _{z \in \mathbb{D}}\left(1-|z|^{2}\right)^{\alpha}\left|\left(\varphi^{\prime}(z)\right)^{2}+\varphi^{\prime \prime}(z) \varphi(z)\right|<\infty .
\end{gathered}
$$

Из (6), (7) и ограниченности функции $\varphi(z)$ имеем

$$
\sup _{z \in \mathbb{D}}\left(1-|z|^{2}\right)^{\alpha}\left|\varphi^{\prime}(z)\right|^{2}<\infty .
$$

Для $w \in \mathbb{D}$ и $b>(\gamma+1) / q$ положим

$$
f_{w}(z)=\frac{\left(1-|w|^{2}\right)^{b-(\gamma+1) / q}}{(1-\bar{w} z)^{1 / p+b}}
$$

Из [16; теорема 1.4.10] известно, что

$$
M_{p}\left(f_{w}, r\right) \leqslant C \frac{\left(1-|w|^{2}\right)^{b-(\gamma+1) / q}}{(1-r|w|)^{b}} .
$$

Из леммы 3 следует, что

$$
\left\|f_{w}\right\|_{H_{p, q, \gamma}}^{q}=\int_{0}^{1} M_{p}^{q}\left(f_{w}, r\right)(1-r)^{\gamma} d r \leqslant C \int_{0}^{1} \frac{\left(1-|w|^{2}\right)^{q b-(\gamma+1)}}{(1-r|w|)^{q b}}(1-r)^{\gamma} d r \leqslant C .
$$

Значит, $f_{w} \in H_{p, q, \gamma}$ и, кроме того, $\sup _{w \in \mathbb{D}}\left\|f_{w}\right\|_{H_{p, q, \gamma}}<\infty$. С другой стороны,

$$
\begin{aligned}
\left|f_{w}^{\prime}(w)\right| & =\left(\frac{1}{p}+b\right) \frac{|w|}{\left(1-|w|^{2}\right)^{(\gamma+1) / q+1 / p+1}} \\
\left|f_{w}^{\prime \prime}(w)\right| & =\left(\frac{1}{p}+b\right)\left(\frac{1}{p}+b+1\right) \frac{|w|^{2}}{\left(1-|w|^{2}\right)^{(\gamma+1) / q+1 / p+2}} .
\end{aligned}
$$


Следовательно,

$$
\begin{aligned}
& C\left\|D C_{\varphi}\right\|_{H_{p, q, \gamma} \rightarrow \mathscr{B}^{\alpha}} \geqslant\left\|D C_{\varphi} f_{\varphi(\lambda)}\right\|_{\mathscr{B}^{\alpha}} \\
& \geqslant-\left(\frac{1}{p}+b\right)\left(\frac{1}{p}+b+1\right) \frac{\left(1-|\lambda|^{2}\right)^{\alpha}\left|\varphi^{\prime}(\lambda)\right|^{2}|\varphi(\lambda)|^{2}}{\left(1-|\varphi(\lambda)|^{2}\right)^{(\gamma+1) / q+1 / p+2}} \\
&+\left(\frac{1}{p}+b\right) \frac{\left(1-|\lambda|^{2}\right)^{\alpha}\left|\varphi^{\prime \prime}(\lambda)\right||\varphi(\lambda)|}{\left(1-|\varphi(\lambda)|^{2}\right)^{(\gamma+1) / q+1 / p+1}}
\end{aligned}
$$

при $\lambda \in \mathbb{D}$. Итак,

$$
\begin{aligned}
& \frac{\left(1-|\lambda|^{2}\right)^{\alpha}\left|\varphi^{\prime \prime}(\lambda)\right||\varphi(\lambda)|}{\left(1-|\varphi(\lambda)|^{2}\right)^{(\gamma+1) / q+1 / p+1}} \\
& \quad \leqslant C\left\|D C_{\varphi}\right\|_{H_{p, q, \gamma} \rightarrow \mathscr{B}^{\alpha}}+\left(\frac{1}{p}+b+1\right) \frac{\left(1-|\lambda|^{2}\right)^{\alpha}\left|\varphi^{\prime}(\lambda)\right|^{2}|\varphi(\lambda)|^{2}}{\left(1-|\varphi(\lambda)|^{2}\right)^{(\gamma+1) / q+1 / p+2}}
\end{aligned}
$$

Далее, при $\lambda \in \mathbb{D}$ положим

$$
g_{\lambda}(z)=\frac{\left(1-|\varphi(\lambda)|^{2}\right)^{b+1-(\gamma+1) / q}}{(1-\overline{\varphi(\lambda)} z)^{1 / p+b+1}}-\frac{1 / p+b+1}{1 / p+b} \frac{\left(1-|\varphi(\lambda)|^{2}\right)^{b-(\gamma+1) / q}}{(1-\overline{\varphi(\lambda)} z)^{1 / p+b}} .
$$

Тогда, как и выше, $\sup _{\lambda \in \mathbb{D}}\left\|g_{\lambda}\right\|_{H_{p, q, \gamma}}<\infty, g_{\lambda}^{\prime}(\varphi(\lambda))=0$ и

$$
\left|g_{\lambda}^{\prime \prime}(\varphi(\lambda))\right|=\left(\frac{1}{p}+b+1\right) \frac{|\varphi(\lambda)|^{2}}{\left(1-|\varphi(\lambda)|^{2}\right)^{(\gamma+1) / q+1 / p+2}} .
$$

Следовательно,

$$
\begin{aligned}
\infty & >C\left\|D C_{\varphi}\right\|_{H_{p, q, \gamma} \rightarrow \mathscr{B}^{\alpha}} \geqslant\left\|D C_{\varphi} g_{\lambda}\right\|_{\mathscr{B}^{\alpha}} \\
& \geqslant\left(\frac{1}{p}+b+1\right) \frac{\left(1-|\lambda|^{2}\right)^{\alpha}\left|\varphi^{\prime}(\lambda)\right|^{2}|\varphi(\lambda)|^{2}}{\left(1-|\varphi(\lambda)|^{2}\right)^{(\gamma+1) / q+1 / p+2}} .
\end{aligned}
$$

Таким образом,

$$
\begin{aligned}
& \sup _{|\varphi(\lambda)|>1 / 2} \frac{\left(1-|\lambda|^{2}\right)^{\alpha}\left|\varphi^{\prime}(\lambda)\right|^{2}}{\left(1-|\varphi(\lambda)|^{2}\right)^{(\gamma+1) / q+1 / p+2}} \leqslant \sup _{|\varphi(\lambda)|>1 / 2} 4 \frac{\left(1-|\lambda|^{2}\right)^{\alpha}\left|\varphi^{\prime}(\lambda)\right|^{2}|\varphi(\lambda)|^{2}}{\left(1-|\varphi(\lambda)|^{2}\right)^{(\gamma+1) / q+1 / p+2}} \\
& \quad \leqslant \sup _{|\varphi(\lambda)|>1 / 2} C\left\|D C_{\varphi}\right\|_{H_{p, q, \gamma} \rightarrow \mathscr{B}^{\alpha}}<\infty .
\end{aligned}
$$

Из равенства (8) имеем

$$
\begin{aligned}
& \sup _{|\varphi(\lambda)| \leqslant 1 / 2} \frac{\left(1-|\lambda|^{2}\right)^{\alpha}\left|\varphi^{\prime}(\lambda)\right|^{2}}{\left(1-|\varphi(\lambda)|^{2}\right)^{(\gamma+1) / q+1 / p+2}} \\
& \quad \leqslant \sup _{|\varphi(\lambda)| \leqslant 1 / 2} \frac{4^{(\gamma+1) / q+1 / p+2}}{3^{(\gamma+1) / q+1 / p+2}}\left(1-|\lambda|^{2}\right)^{\alpha}\left|\varphi^{\prime}(\lambda)\right|^{2}<\infty .
\end{aligned}
$$

В силу (10) и (11) получаем неравенство

$$
\sup _{\lambda \in \mathbb{D}} \frac{\left(1-|\lambda|^{2}\right)^{\alpha}\left|\varphi^{\prime}(\lambda)\right|^{2}}{\left(1-|\varphi(\lambda)|^{2}\right)^{(\gamma+1) / q+1 / p+2}}<\infty .
$$


Из (9) следует, что

$$
\sup _{\lambda \in \mathbb{D}} \frac{\left(1-|\lambda|^{2}\right)^{\alpha}\left|\varphi^{\prime \prime}(\lambda)\right||\varphi(\lambda)|}{\left(1-|\varphi(\lambda)|^{2}\right)^{(\gamma+1) / q+1 / p+1}}<\infty .
$$

Теперь (12) и (6) дают

$$
\begin{aligned}
& \sup _{|\varphi(\lambda)|>1 / 2} \frac{\left(1-|\lambda|^{2}\right)^{\alpha}\left|\varphi^{\prime \prime}(\lambda)\right|}{\left(1-|\varphi(\lambda)|^{2}\right)^{(\gamma+1) / q+1 / p+1}} \\
& \leqslant 2 \sup _{|\varphi(\lambda)|>1 / 2} \frac{\left(1-|\lambda|^{2}\right)^{\alpha}\left|\varphi^{\prime \prime}(\lambda)\right||\varphi(\lambda)|}{\left(1-|\varphi(\lambda)|^{2}\right)^{(\gamma+1) / q+1 / p+1}}<\infty, \\
& \sup _{|\varphi(\lambda)| \leqslant 1 / 2} \frac{\left(1-|\lambda|^{2}\right)^{\alpha}\left|\varphi^{\prime \prime}(\lambda)\right|}{\left(1-|\varphi(\lambda)|^{2}\right)^{(\gamma+1) / q+1 / p+1}} \\
& \leqslant \frac{4^{(\gamma+1) / q+1 / p+1}}{3^{(\gamma+1) / q+1 / p+1}} \sup _{|\varphi(\lambda)| \leqslant 1 / 2}\left(1-|\lambda|^{2}\right)^{\alpha}\left|\varphi^{\prime \prime}(\lambda)\right|<\infty .
\end{aligned}
$$

Объединяя (13) и (14), получаем (4). Доказательство теоремы 1 завершено.

Теорема 2. Пусть $0<p, q, \alpha<\infty,-1<\gamma<\infty u \varphi-$ аналитическое отображение $\mathbb{D}$ в себя. Тогда оператор $D C_{\varphi}: H_{p, q, \gamma} \rightarrow \mathscr{B}^{\alpha}$ компактен тогда и только тогда, когда он ограничен и

$$
\begin{gathered}
\lim _{|\varphi(z)| \rightarrow 1} \frac{\left|\varphi^{\prime}(z)\right|^{2}\left(1-|z|^{2}\right)^{\alpha}}{\left(1-|\varphi(z)|^{2}\right)^{(\gamma+1) / q+1 / p+2}}=0, \\
\lim _{|\varphi(z)| \rightarrow 1} \frac{\left|\varphi^{\prime \prime}(z)\right|\left(1-|z|^{2}\right)^{\alpha}}{\left(1-|\varphi(z)|^{2}\right)^{(\gamma+1) / q+1 / p+1}}=0 .
\end{gathered}
$$

ДоказАтельство. Достаточность. По условию для всякого $\varepsilon>0$ существует $\delta \in(0,1)$, при котором из неравенства $\delta<|\varphi(z)|<1$ следует, что

$$
\frac{\left|\varphi^{\prime}(z)\right|^{2}\left(1-|z|^{2}\right)^{\alpha}}{\left(1-|\varphi(z)|^{2}\right)^{(\gamma+1) / q+1 / p+2}}<\varepsilon, \quad \frac{\left|\varphi^{\prime \prime}(z)\right|\left(1-|z|^{2}\right)^{\alpha}}{\left(1-|\varphi(z)|^{2}\right)^{(\gamma+1) / q+1 / p+1}}<\varepsilon .
$$

Так как оператор $D C_{\varphi}: H_{p, q, \gamma} \rightarrow \mathscr{B}^{\alpha}$ ограничен, то из доказательства теоремы 1 следует, что

$$
M_{1}=\sup _{z \in \mathbb{D}}\left|\varphi^{\prime \prime}(z)\right|\left(1-|z|^{2}\right)^{\alpha}<\infty, \quad M_{2}=\sup _{z \in \mathbb{D}}\left|\varphi^{\prime}(z)\right|^{2}\left(1-|z|^{2}\right)^{\alpha}<\infty .
$$

Пусть $\left(f_{k}\right)_{k \in \mathbb{N}}$ - такая последовательность из $H_{p, q, \gamma}$, для которой

$$
\sup _{k \in \mathbb{N}}\left\|f_{k}\right\|_{H_{p, q, \gamma}}<\infty
$$

и $f_{k} \rightarrow 0, k \rightarrow \infty$, равномерно внутри $\mathbb{D}$. Пусть $K=\{z \in \mathbb{D}:|\varphi(z)| \leqslant \delta\}$. Тогда имеем

$$
\begin{gathered}
\left\|D C_{\varphi} f_{k}\right\|_{\mathscr{B}^{\alpha}}=\sup _{z \in \mathbb{D}}\left|\left(D C_{\varphi} f_{k}\right)^{\prime}(z)\right|\left(1-|z|^{2}\right)^{\alpha}+\left|f_{k}^{\prime}(\varphi(0)) \varphi^{\prime}(0)\right| \\
\leqslant \sup _{z \in \mathbb{D}}\left|\left(\varphi^{\prime} f_{k}^{\prime}(\varphi)\right)^{\prime}(z)\right|\left(1-|z|^{2}\right)^{\alpha}+\left|f_{k}^{\prime}(\varphi(0)) \varphi^{\prime}(0)\right|
\end{gathered}
$$




$$
\begin{aligned}
& \leqslant \sup _{z \in \mathbb{D}}\left(1-|z|^{2}\right)^{\alpha}\left|\varphi^{\prime}(z)\right|^{2}\left|f_{k}^{\prime \prime}(\varphi(z))\right| \\
& +\sup _{z \in \mathbb{D}}\left(1-|z|^{2}\right)^{\alpha}\left|\varphi^{\prime \prime}(z)\right|\left|f_{k}^{\prime}(\varphi(z))\right|+\left|f_{k}^{\prime}(\varphi(0)) \varphi^{\prime}(0)\right| \\
& \leqslant \sup _{z \in K}\left(1-|z|^{2}\right)^{\alpha}\left|\varphi^{\prime}(z)\right|^{2}\left|f_{k}^{\prime \prime}(\varphi(z))\right|+\sup _{z \in K}\left(1-|z|^{2}\right)^{\alpha}\left|\varphi^{\prime \prime}(z)\right|\left|f_{k}^{\prime}(\varphi(z))\right| \\
& +\sup _{z \in \mathbb{D} \backslash K}\left(1-|z|^{2}\right)^{\alpha}\left|\varphi^{\prime}(z)\right|^{2}\left|f_{k}^{\prime \prime}(\varphi(z))\right| \\
& +\sup _{z \in \mathbb{D} \backslash K}\left(1-|z|^{2}\right)^{\alpha}\left|\varphi^{\prime \prime}(z)\right|\left|f_{k}^{\prime}(\varphi(z))\right|+\left|f_{k}^{\prime}(\varphi(0)) \varphi^{\prime}(0)\right| \\
& \leqslant \sup _{z \in K}\left(1-|z|^{2}\right)^{\alpha}\left|\varphi^{\prime}(z)\right|^{2}\left|f_{k}^{\prime \prime}(\varphi(z))\right|+\sup _{z \in K}\left(1-|z|^{2}\right)^{\alpha}\left|\varphi^{\prime \prime}(z)\right|\left|f_{k}^{\prime}(\varphi(z))\right| \\
& +\left|f_{k}^{\prime}(\varphi(0)) \varphi^{\prime}(0)\right|+C \sup _{z \in \mathbb{D} \backslash K} \frac{\left|\varphi^{\prime}(z)\right|^{2}\left(1-|z|^{2}\right)^{\alpha}}{\left(1-|\varphi(z)|^{2}\right)^{(\gamma+1) / q+1 / p+2}}\left\|f_{k}\right\|_{H_{p, q, \gamma}} \\
& +C \sup _{z \in \mathbb{D} \backslash K} \frac{\left|\varphi^{\prime \prime}(z)\right|\left(1-|z|^{2}\right)^{\alpha}}{\left(1-|\varphi(z)|^{2}\right)^{(\gamma+1) / q+1 / p+1}}\left\|f_{k}\right\|_{H_{p, q, \gamma}} \\
& \leqslant M_{2} \sup _{|\zeta| \leqslant \delta}\left|f_{k}^{\prime \prime}(\zeta)\right|+M_{1} \sup _{|\zeta| \leqslant \delta}\left|f_{k}^{\prime}(\zeta)\right|+2 C \varepsilon\left\|f_{k}\right\|_{H_{p, q, \gamma}}+\left|f_{k}^{\prime}(\varphi(0)) \varphi^{\prime}(0)\right| \text {. }
\end{aligned}
$$

Из предположения о сходимости $f_{k} \rightarrow 0, k \rightarrow \infty$, внутри $\mathbb{D}$ и неравенств Коши следует, что $f_{k}^{\prime} \rightarrow 0$ и $f_{k}^{\prime \prime} \rightarrow 0, k \rightarrow \infty$, внутри $\mathbb{D}$. Значит, переходя к пределу в (16) при $k \rightarrow \infty$ и используя произвольность $\varepsilon>0$, получаем, что

$$
\lim _{k \rightarrow \infty}\left\|D C_{\varphi} f_{k}\right\|_{\mathscr{B}^{\alpha}}=0
$$

Применение леммы 4 доказывает требуемое утверждение.

Необходимость. Пусть $D C_{\varphi}: H_{p, q, \gamma} \rightarrow \mathscr{B}^{\alpha}-$ компактный оператор. Тогда он ограничен. Пусть $\left(z_{k}\right)_{k \in \mathbb{N}}-$ последовательность точек из $\mathbb{D}$, по которой $\left|\varphi\left(z_{k}\right)\right| \rightarrow 1, k \rightarrow \infty$. Пусть

$$
f_{k}(z)=\frac{\left(1-\left|\varphi\left(z_{k}\right)\right|^{2}\right)^{b-(\gamma+1) / q}}{\left(1-\overline{\varphi\left(z_{k}\right)} z\right)^{1 / p+b}}, \quad k \in \mathbb{N}
$$

где $b>(\gamma+1) / q$. Тогда $f_{k} \in H_{p, q, \gamma}$ и, более того, $\sup _{k \in \mathbb{N}}\left\|f_{k}\right\|_{H_{p, q, \gamma}}<\infty$, а $f_{k} \rightarrow 0, k \rightarrow \infty$, равномерно внутри $\mathbb{D}$. Поскольку оператор $D C_{\varphi}: H_{p, q, \gamma} \rightarrow \mathscr{B}^{\alpha}$ компактен, то по лемме 4 получаем

$$
\lim _{k \rightarrow \infty}\left\|D C_{\varphi} f_{k}\right\|_{\mathscr{B}^{\alpha}}=0
$$

Аналогично доказательству теоремы 1 имеем

$$
\begin{gathered}
\left\|D C_{\varphi} f_{k}\right\|_{\mathscr{B}^{\alpha}} \geqslant \mid-\left(b+\frac{1}{p}\right)\left(b+\frac{1}{p}+1\right) \frac{\left(1-\left|z_{k}\right|^{2}\right)^{\alpha}\left|\varphi^{\prime}\left(z_{k}\right)\right|^{2}\left|\varphi\left(z_{k}\right)\right|^{2}}{\left(1-\left|\varphi\left(z_{k}\right)\right|^{2}\right)^{(\gamma+1) / q+1 / p+2}} \\
+\left(b+\frac{1}{p}\right) \frac{\left(1-\left|z_{k}\right|^{2}\right)^{\alpha}\left|\varphi^{\prime \prime}\left(z_{k}\right)\right|\left|\varphi\left(z_{k}\right)\right|}{\left(1-\left|\varphi\left(z_{k}\right)\right|^{2}\right)^{(\gamma+1) / q+1 / p+1} \mid}
\end{gathered}
$$


откуда следует, что

$$
\begin{gathered}
\lim _{k \rightarrow \infty}\left(b+\frac{1}{p}+1\right) \frac{\left(1-\left|z_{k}\right|^{2}\right)^{\alpha}\left|\varphi^{\prime}\left(z_{k}\right)\right|^{2}\left|\varphi\left(z_{k}\right)\right|^{2}}{\left(1-\left|\varphi\left(z_{k}\right)\right|^{2}\right)^{(\gamma+1) / q+1 / p+2}} \\
\quad=\lim _{k \rightarrow \infty} \frac{\left(1-\left|z_{k}\right|^{2}\right)^{\alpha}\left|\varphi^{\prime \prime}\left(z_{k}\right)\right|\left|\varphi\left(z_{k}\right)\right|}{\left(1-\left|\varphi\left(z_{k}\right)\right|^{2}\right)^{(\gamma+1) / q+1 / p+1}}
\end{gathered}
$$

если хотя бы один из пределов определен.

Положим теперь

$$
g_{k}(z)=\frac{\left(1-\left|\varphi\left(z_{k}\right)\right|^{2}\right)^{b+1-(\gamma+1) / q}}{\left(1-\overline{\varphi\left(z_{k}\right)} z\right)^{1 / p+b+1}}-\frac{1 / p+b+1}{1 / p+b} \frac{\left(1-\left|\varphi\left(z_{k}\right)\right|^{2}\right)^{b-(\gamma+1) / q}}{\left(1-\overline{\varphi\left(z_{k}\right)} z\right)^{1 / p+b}}, \quad k \in \mathbb{N},
$$

для последовательности $\left(z_{k}\right)_{k \in \mathbb{N}} \in \mathbb{D}$, по которой $\left|\varphi\left(z_{k}\right)\right| \rightarrow 1, k \rightarrow \infty$. Тогда $\left(g_{k}\right)_{k \in \mathbb{N}}$ - ограниченная последовательность в $H_{p, q, \gamma}$ и $g_{k} \rightarrow 0, k \rightarrow \infty$, равномерно внутри $\mathbb{D}$. Заметим также, что $g_{k}^{\prime}\left(\varphi\left(z_{k}\right)\right)=0$ и

$$
\left|g_{k}^{\prime \prime}\left(\varphi\left(z_{k}\right)\right)\right|=\frac{(1 / p+b+1)\left|\varphi\left(z_{k}\right)\right|^{2}}{\left(1-\left|\varphi\left(z_{k}\right)\right|^{2}\right)^{(\gamma+1) / q+1 / p+2}} .
$$

Поскольку $D C_{\varphi}: H_{p, q, \gamma} \rightarrow \mathscr{B}^{\alpha}-$ компактный оператор, то имеем

$$
\lim _{k \rightarrow \infty}\left\|D C_{\varphi} g_{k}\right\|_{\mathscr{B}^{\alpha}}=0 .
$$

С другой стороны,

$$
\frac{(1 / p+b+1)\left(1-\left|z_{k}\right|^{2}\right)^{\alpha}\left|\varphi^{\prime}\left(z_{k}\right)\right|^{2}\left|\varphi\left(z_{k}\right)\right|^{2}}{\left(1-\left|\varphi\left(z_{k}\right)\right|^{2}\right)^{(\gamma+1) / q+1 / p+2}} \leqslant\left\|D C_{\varphi} g_{k}\right\|_{\mathscr{B}^{\alpha}} .
$$

Таким образом,

$$
\lim _{k \rightarrow \infty} \frac{\left(1-\left|z_{k}\right|^{2}\right)^{\alpha}\left|\varphi^{\prime}\left(z_{k}\right)\right|^{2}}{\left(1-\left|\varphi\left(z_{k}\right)\right|^{2}\right)^{(\gamma+1) / q+1 / p+2}}=\lim _{k \rightarrow \infty} \frac{\left(1-\left|z_{k}\right|^{2}\right)^{\alpha}\left|\varphi^{\prime}\left(z_{k}\right)\right|^{2}\left|\varphi\left(z_{k}\right)\right|^{2}}{\left(1-\left|\varphi\left(z_{k}\right)\right|^{2}\right)^{(\gamma+1) / q+1 / p+2}}=0 .
$$

Теперь из этого равенства и формулы (18) следует, что

$$
\lim _{k \rightarrow \infty} \frac{\left(1-\left|z_{k}\right|^{2}\right)^{\alpha}\left|\varphi^{\prime \prime}\left(z_{k}\right)\right|}{\left(1-\left|\varphi\left(z_{k}\right)\right|^{2}\right)^{(\gamma+1) / q+1 / p+1}}=0 .
$$

Требуемый результат вытекает из двух последних соотношений.

\section{§ 3. Ограниченность и компактность оператора $D C_{\varphi}: H_{p, q, \gamma} \rightarrow \mathscr{B}_{0}^{\alpha}$}

В этом параграфе мы охарактеризуем свойства ограниченности и компактности оператора $D C_{\varphi}: H_{p, q, \gamma} \rightarrow \mathscr{B}_{0}^{\alpha}$. Для этого нам потребуется следующая лемма (см. [18]).

Лемма 5. Пусть $\alpha>0$. Замкнутое множество $K$ в $\mathscr{B}_{0}^{\alpha}$ компактно в том и только том случае, когда оно ограничено и выполнено условие

$$
\lim _{|z| \rightarrow 1} \sup _{f \in K}\left(1-|z|^{2}\right)^{\alpha}\left|f^{\prime}(z)\right|=0 .
$$


Теорема 3. Пусть $0<p, q, \alpha<\infty,-1<\gamma<\infty u \varphi-$ аналитическое отображение $\mathbb{D}$ в себя. Тогда оператор $D C_{\varphi}: H_{p, q, \gamma} \rightarrow \mathscr{B}_{0}^{\alpha}$ ограничен в том и только том случае, когда оператор $D C_{\varphi}: H_{p, q, \gamma} \rightarrow \mathscr{B}^{\alpha}$ ограничен $u$

$$
\lim _{|z| \rightarrow 1}\left(1-|z|^{2}\right)^{\alpha}\left|\varphi^{\prime \prime}(z)\right|=0, \quad \lim _{|z| \rightarrow 1}\left(1-|z|^{2}\right)^{\alpha}\left|\varphi^{\prime}(z)\right|^{2}=0 .
$$

ДоказАтельство. Предположим сначала, что $D C_{\varphi}: H_{p, q, \gamma} \rightarrow \mathscr{B}_{0}^{\alpha}$ ограничен. Тогда ясно, что и $D C_{\varphi}: H_{p, q, \gamma} \rightarrow \mathscr{B}^{\alpha}$ ограничен. Рассматривая $f(z)=z$ и $f(z)=z^{2}$, получаем (19).

Пусть, наоборот, $D C_{\varphi}: H_{p, q, \gamma} \rightarrow \mathscr{B}^{\alpha}$ ограничен и выполнено (19). Для всякого полинома $p$ получаем

$$
\begin{aligned}
& \left(1-|z|^{2}\right)^{\alpha}\left|\left(D C_{\varphi} p\right)^{\prime}(z)\right| \\
& \quad \leqslant\left(1-|z|^{2}\right)^{\alpha}\left|\varphi^{\prime}(z)\right|^{2}\left|p^{\prime \prime}(\varphi(z))\right|+\left(1-|z|^{2}\right)^{\alpha}\left|\varphi^{\prime \prime}(z) p^{\prime}(\varphi(z))\right| .
\end{aligned}
$$

Поскольку

$$
\sup _{w \in \mathbb{D}}\left|p^{\prime \prime}(w)\right|<\infty, \quad \sup _{w \in \mathbb{D}}\left|p^{\prime}(w)\right|<\infty
$$

и выполнено $(20)$, то $D C_{\varphi} p \in \mathscr{B}_{0}^{\alpha}$. Так как полиномы плотны в $H_{p, q, \gamma}$, то для всякого $f \in H_{p, q, \gamma}$ существует последовательность полиномов $\left(p_{n}\right)_{n \in \mathbb{N}}$, для которой $\left\|f-p_{n}\right\|_{H_{p, q, \gamma}} \rightarrow 0, n \rightarrow \infty$. Значит,

$$
\left\|D C_{\varphi} f-D C_{\varphi} p_{n}\right\|_{\mathscr{B}^{\alpha}} \leqslant\left\|D C_{\varphi}\right\|_{H_{p, q, \gamma} \rightarrow \mathscr{B}^{\alpha}}\left\|f-p_{n}\right\|_{H_{p, q, \gamma}} \rightarrow 0, \quad n \rightarrow \infty,
$$

так как $D C_{\varphi}: H_{p, q, \gamma} \rightarrow \mathscr{B}^{\alpha}$ - ограниченный оператор. Поскольку $\mathscr{B}_{0}^{\alpha}-$ замкнутое подмножество $\mathscr{B}^{\alpha}$, то получаем, что

$$
D C_{\varphi}\left(H_{p, q, \gamma}\right) \subset \mathscr{B}_{0}^{\alpha} .
$$

Значит, $D C_{\varphi}: H_{p, q, \gamma} \rightarrow \mathscr{B}_{0}^{\alpha}-$ ограниченный оператор.

Tеорема 4. Пусть $0<p, q, \alpha<\infty,-1<\gamma<\infty u \varphi$ - аналитическое отображение $\mathbb{D}$ в себя. Тогда оператор $D C_{\varphi}: H_{p, q, \gamma} \rightarrow \mathscr{B}_{0}^{\alpha}$ компактен в том $u$ только том случае, когда

$$
\begin{aligned}
& \lim _{|z| \rightarrow 1} \frac{\left(1-|z|^{2}\right)^{\alpha}\left|\varphi^{\prime}(z)\right|^{2}}{\left(1-|\varphi(z)|^{2}\right)^{(\gamma+1) / q+1 / p+2}}=0, \\
& \lim _{|z| \rightarrow 1} \frac{\left(1-|z|^{2}\right)^{\alpha}\left|\varphi^{\prime \prime}(z)\right|}{\left(1-|\varphi(z)|^{2}\right)^{(\gamma+1) / q+1 / p+1}}=0 .
\end{aligned}
$$

ДоказАТЕЛьство. Достаточность. Пусть $f \in H_{p, q, \gamma}$. Тогда

$$
\begin{aligned}
& \left(1-|z|^{2}\right)^{\alpha}\left|\left(D C_{\varphi} f\right)^{\prime}(z)\right| \\
& \quad \leqslant C\left(\frac{\left(1-|z|^{2}\right)^{\alpha}\left|\varphi^{\prime}(z)\right|^{2}}{\left(1-|\varphi(z)|^{2}\right)^{(\gamma+1) / q+1 / p+2}}+\frac{\left(1-|z|^{2}\right)^{\alpha}\left|\varphi^{\prime \prime}(z)\right|}{\left(1-|\varphi(z)|^{2}\right)^{(\gamma+1) / q+1 / p+1}}\right)\|f\|_{H_{p, q, \gamma}} .
\end{aligned}
$$

Взяв здесь верхнюю грань по всем $f \in H_{p, q, \gamma}$ таким, что $\|f\|_{H_{p, q, \gamma}} \leqslant 1$, и переходя к пределу при $|z| \rightarrow 1$, получаем

$$
\lim _{|z| \rightarrow 1} \sup _{\|f\|_{H_{p, q, \gamma}} \leqslant 1}\left(1-|z|^{2}\right)^{\alpha}\left|\left(D C_{\varphi} f\right)^{\prime}(z)\right|=0 .
$$

Отсюда по лемме 5 оператор $D C_{\varphi}: H_{p, q, \gamma} \rightarrow \mathscr{B}_{0}^{\alpha}$ компактен. 
Необходимость. Предположим, что $D C_{\varphi}: H_{p, q, \gamma} \rightarrow \mathscr{B}_{0}^{\alpha}$ компактен. Тогда ясно, что $D C_{\varphi}: H_{p, q, \gamma} \rightarrow \mathscr{B}_{0}^{\alpha}$ ограничен. Взяв функцию $f$ вида $f(z)=z$, получаем

$$
\lim _{|z| \rightarrow 1}\left(1-|z|^{2}\right)^{\alpha}\left|\varphi^{\prime \prime}(z)\right|=0
$$

Теперь, взяв $f(z)=z^{2}$ и используя ограниченность оператора $D C_{\varphi}: H_{p, q, \gamma} \rightarrow$ $\mathscr{B}_{0}^{\alpha}$, видим, что

$$
\lim _{|z| \rightarrow 1}\left(1-|z|^{2}\right)^{\alpha}\left|\varphi^{\prime}(z)\right|^{2}=0 .
$$

Если $\|\varphi\|_{\infty}<1$, то из $(23)$ и (24) получаем

$$
\begin{aligned}
& \lim _{|z| \rightarrow 1} \frac{\left(1-|z|^{2}\right)^{\alpha}\left|\varphi^{\prime}(z)\right|^{2}}{\left(1-|\varphi(z)|^{2}\right)^{(\gamma+1) / q+1 / p+2}} \\
& \quad \leqslant \frac{1}{\left(1-\|\varphi\|_{\infty}^{2}\right)^{(\gamma+1) / q+1 / p+2}} \lim _{|z| \rightarrow 1}\left(1-|z|^{2}\right)^{\alpha}\left|\varphi^{\prime}(z)\right|^{2}=0, \\
& \lim _{|z| \rightarrow 1} \frac{\left(1-|z|^{2}\right)^{\alpha}\left|\varphi^{\prime \prime}(z)\right|}{\left(1-|\varphi(z)|^{2}\right)^{(\gamma+1) / q+1 / p+1}} \\
& \quad \leqslant \frac{1}{\left(1-\|\varphi\|_{\infty}^{2}\right)^{(\gamma+1) / q+1 / p+1}} \lim _{|z| \rightarrow 1}\left(1-|z|^{2}\right)^{\alpha}\left|\varphi^{\prime \prime}(z)\right|=0,
\end{aligned}
$$

что и доказывает утверждение теоремы в этом случае.

Предположим теперь, что $\|\varphi\|_{\infty}=1$, и пусть $\left(\varphi\left(z_{k}\right)\right)_{k \in \mathbb{N}}-$ такая последовательность, что

$$
\lim _{k \rightarrow \infty}\left|\varphi\left(z_{k}\right)\right|=1
$$

Положим для $k \in \mathbb{N}$

$$
\begin{gathered}
f_{k}(z)=\frac{\left(1-\left|\varphi\left(z_{k}\right)\right|^{2}\right)^{b-(\gamma+1) / q}}{\left(1-\overline{\varphi\left(z_{k}\right)} z\right)^{1 / p+b}}, \\
g_{k}(z)=\frac{\left(1-\left|\varphi\left(z_{k}\right)\right|^{2}\right)^{b+1-(\gamma+1) / q}}{\left(1-\overline{\varphi\left(z_{k}\right)} z\right)^{1 / p+b+1}}-\frac{1 / p+b+1}{1 / p+b} \frac{\left(1-\left|\varphi\left(z_{k}\right)\right|^{2}\right)^{b-(\gamma+1) / q}}{\left(1-\overline{\varphi\left(z_{k}\right)} z\right)^{1 / p+b}} .
\end{gathered}
$$

В силу доказательства теоремы 2 имеем

$$
\begin{gathered}
\lim _{|\varphi(z)| \rightarrow 1} \frac{\left(1-|z|^{2}\right)^{\alpha}\left|\varphi^{\prime}(z)\right|^{2}}{\left(1-|\varphi(z)|^{2}\right)^{(\gamma+1) / q+1 / p+2}}=0, \\
\lim _{|\varphi(z)| \rightarrow 1} \frac{\left(1-|z|^{2}\right)^{\alpha}\left|\varphi^{\prime \prime}(z)\right|}{\left(1-|\varphi(z)|^{2}\right)^{(\gamma+1) / q+1 / p+1}}=0 .
\end{gathered}
$$

Из (23) и (26) следует, что для любого $\varepsilon>0$ существует такое $r \in(0,1)$, что

$$
\frac{\left(1-|z|^{2}\right)^{\alpha}\left|\varphi^{\prime \prime}(z)\right|}{\left(1-|\varphi(z)|^{2}\right)^{(\gamma+1) / q+1 / p+1}}<\varepsilon
$$

при $r<|\varphi(z)|<1$, и существует такое $\sigma \in(0,1)$, что

$$
\left(1-|z|^{2}\right)^{\alpha}\left|\varphi^{\prime \prime}(z)\right| \leqslant \varepsilon\left(1-r^{2}\right)^{(\gamma+1) / q+1 / p+1}
$$


при $\sigma<|z|<1$. Значит, при $\sigma<|z|<1$ и $r<|\varphi(z)|<1$ имеем

$$
\frac{\left(1-|z|^{2}\right)^{\alpha}\left|\varphi^{\prime \prime}(z)\right|}{\left(1-|\varphi(z)|^{2}\right)^{(\gamma+1) / q+1 / p+1}}<\varepsilon .
$$

С другой стороны, если $\sigma<|z|<1$ и $|\varphi(z)| \leqslant r$, то из (27) получаем

$$
\frac{\left(1-|z|^{2}\right)^{\alpha}\left|\varphi^{\prime \prime}(z)\right|}{\left(1-|\varphi(z)|^{2}\right)^{(\gamma+1) / q+1 / p+1}}<\frac{1}{\left(1-r^{2}\right)^{(\gamma+1) / q+1 / p+1}}\left(1-|z|^{2}\right)^{\alpha}\left|\varphi^{\prime \prime}(z)\right|<\varepsilon
$$

Объединение (28) и (29) приводит к (22), а формула (21) выводится из (24) и (25) аналогично тому, как это было сделано выше. Теорема 4 доказана.

Благодарности. Исследования Сонгсиао Ли были частично поддержаны Китайским государственным фондом естественных наук (№№ 10371051, 10371069).

\section{Список литературы}

[1] C. C. Cowen, B. D. MacCluer, Composition operators on spaces of analytic functions, Stud. Adv. Math., CRC Press, Boca Raton, FL, 1995.

[2] J. H. Shapiro, Composition operators and classical function theory, Universitext Tracts Math., Springer-Verlag, New York, 1993.

[3] K. Zhu, Operator theory in function spaces, Pure Appl. Math., 139, Dekker, New York, 1990.

[4] R. A. Hibschweiler, N. Portnoy, "Composition followed by differentiation between Bergman and Hardy spaces", Rocky Mountain J. Math., 35:3 (2005), 843-855.

[5] D.-Ch. Chang, S. Stević, "The generalized Cesàro operator on the unit polydisk", Taiwanese J. Math., 7:2 (2003), 293-308.

[6] D.-Ch. Chang, S. Stević, "Addendum to the paper "A note on weighted Bergman spaces and the Cesàro operator", Nagoya Math. J., 180 (2005), 77-90.

[7] D. D. Clahane, S. Stević, "Norm equivalence and composition operators between Bloch/Lipschitz spaces of the ball", J. Inequal. Appl., 2006, article ID 61018.

[8] S. Stević, "Composition operators on the generalized Bergman space", J. Indian Math. Soc., 69:1-4 (2002), 61-64.

[9] S. Stević, "Cesàro averaging operators", Math. Nachr., 248-249:1 (2003), 185-189.

[10] S. Stević, "Hilbert operator on the polydisk", Bull. Inst. Math. Acad. Sinica, 31:2 (2003), 135-142.

[11] S. Stević, "The generalized Cesàro operator on Dirichlet spaces", Studia Sci. Math. Hungar., 40:1-2 (2003), 83-94.

[12] S. Stević, "The generalized Libera transform on Hardy, Bergman and Bloch spaces on the unit polydisc", Z. Anal. Anwendungen, 22:1 (2003), 179-186.

[13] S. Stević, "A note on the generalized Cesàro operator on Bergman spaces", Indian J. Math., 46:1 (2004), 129-136.

[14] S. Stević, "On an integral operator on the unit ball in $\mathbb{C}^{n ",}$ J. Inequal. Appl., 2005, № $1,81-88$.

[15] J.-H. Shi, "Inequalities for the integral means of holomorphic functions and their derivatives in the unit ball of $\mathbb{C}^{n}$ ", Trans. Amer. Math. Soc., 328:2 (1991), 619-637.

[16] W. Rudin, Function theory in the unit ball of $\mathbb{C}^{n}$, Grundlehren Math. Wiss., 241, Springer-Verlag, New York-Berlin, 1980; рус. пер.: У. Рудин, Теория функиий в единичном шаре из $\mathbb{C}^{n}$, Мир, М., 1984. 
[17] A. L. Shields, D. L. Williams, "Bounded projections, duality, and multipliers in spaces of analytic functions", Trans. Amer. Math. Soc., 162 (1971), 287-302.

[18] Sh. Ohno, K. Stroethoff, R. Zhao, "Weighted composition operators between Blochtype spaces", Rocky Mountain J. Math., 33:1 (2003), 191-215.

\section{Сонгсиао Ли (Songxiao Li)}

Department of Mathematics,

Jiaying University, Meizhou, China

E-mail: jyulsx@163.com, lsx@mail.zjxu.edu.cn

C. Стевич (S. Stević)

Mathematical Institute,

Serbian Academy of Science and Arts, Beograd, Serbia

E-mail: sstevic@ptt.rs

Перевод с англ.
Поступила в редакцию

03.11 .2006 